\title{
LA DEIXIS PERSONAL EN LA INTERPRETACIÓN SIMULTÁNEA DEL ESPAÑOL AL ITALIANO
}

\author{
Sara Bani \\ sara.bani@unict.it \\ Universidad de Catania
}

\section{Resumen}

La deixis personal codifica el papel de los interlocutores en el evento comunicativo y desempeña un papel fundamental en la construcción retórica del discurso. En la interpretación simultánea, los deícticos personales pueden experimentar diferentes cambios: algunas referencias se omiten, mientras que otras se anaden o se modifican. El objetivo del estudio es analizar el tratamiento de la deixis personal, y más específicamente de la primera persona del singular y del plural, en un corpus de textos interpretados en simultánea del español al italiano. Todos los textos del corpus fueron pronunciados por conferenciantes latinoamericanos en el ámbito del festival de periodismo de la revista Internazionale y fueron interpretados simultáneamente al italiano por intérpretes profesionales. El estudio pretende identificar tendencias en el tratamiento de los deícticos personales en la interpretación simultánea del español al italiano.

\begin{abstract}
"Personal deixis in Spanish-Italian simultaneous interpretation"

Personal deixis encodes the role of the participants in a communicative event and it is relevant to the rhetorical construction of discourse. In simultaneous interpretation, personal deixis may undergo significant changes: some references are omitted, while others are added or modified. The study examines how first person singular and plural deixis is interpreted from Spanish into Italian. The analysis is based on a corpus of spoken texts which were pronounced by Latin American speakers during a journalism festival organized by the magazine Internazionale. All texts were simultaneously interpreted from Spanish into Italian by freelance professional interpreters. The aim of this study is to identify trends in the simultaneous interpretation of personal deixis from Spanish into Italian.
\end{abstract}


Palabras clave: Pronombres personales. Deixis. Periodismo. Interpretación españolitaliano. Internazionale.

Keywords: Personal pronouns. Deixis. Journalism. Spanish-Italian interpretation. Internazionale.

Manuscript received on April 29, 2015 and accepted for publication on September 19, 2015. 


\section{Introducción y objetivos del estudio}

Uno de los macrorasgos típicos de la oralidad es la presencia de un contexto extralingüístico común (Bazzanella 1994: 14). La deixis es el medio con el que se establece una relación entre la lengua y el contexto extralingüístico, ya que la interpretación de las expresiones deícticas está estrechamente vinculada con el contexto específico de la comunicación. Como indica Eguren (1999: 931), los deícticos son "una clase relativamente cerrada de unidades o expresiones lingüísticas que permiten hacer referencia a un número ilimitado de entidades del mundo".

La Grande grammatica italiana di consultazione (Vanelli \& Renzi 2001: 263) define la deixis como la codificación lingüística de rasgos contextuales vinculados con la organización egocéntrica de la interacción comunicativa. La situación de enunciación canónica es egocéntrica en el sentido de que el hablante, al ser el emisor del mensaje, asume el papel de ego y lo relaciona todo con su punto de vista, con una egocentricidad que es tanto espacial como temporal (Lyons 1977: 638). Por tanto, la interpretación de los deícticos gira alrededor del centro deíctico o punto cero de las coordenadas espacio-temporales del contexto: el yo, el aquí y el ahora (Eguren 1999: 932).

Persona, tiempo y espacio son tres rasgos que constituyen universales de la lengua a nivel conceptual, si bien su manifestación en las categorías gramaticales dista de ser uniforme: la universalidad de las categorías deícticas es independiente de su expresión gramatical, pero de alguna manera siempre se refleja a nivel gramatical o léxico (Levinson 2004: 112).

Las expresiones deícticas han despertado el interés de muchas disciplinas. La filosofía, y más específicamente la lógica, ha destacado que el conocimiento del contexto es crucial para establecer la verdad de una enunciación que contiene una expresión deíctica o, para citar a Lyons (1977: 646), "la deixis establece límites a las posibilidades de decontextualización". Deixis puede ser un sinónimo del término indexicalidad, aunque en algunos casos (como en Levinson 2004: 97) dicho término se utiliza para referirse a los fenómenos más amplios de dependencia contextual, aplicando el término deixis a los fenómenos más relevantes de la indexicalidad desde un punto 
de vista lingüístico. En la lógica se utiliza la expresión campo indical para referirse al sistema de coordenadas espaciales, temporales y personales cuyo centro está representado por el momento y las circunstancias de la enunciación. En el ámbito psicológico, Rommetveit (1968) acuñó la definición de anclaje deíctico para destacar que las expresiones deícticas ponen en relación el enunciado con el contexto espacio-temporal de la enunciación. Esa expresión fue retomada y utilizada en ámbito lingüístico por Fillmore (1975).

Existen muchas clasificaciones de la deixis que están basadas en diferentes criterios y que en algunos casos se solapan. Se atribuye a Fillmore y a Lyons la clasificación más tradicional y difundida de la deixis en personal, espacial y temporal (a las que posteriormente se añadieron la deixis social y la deixis textual).

La deixis personal codifica el papel de los interlocutores en el evento comunicativo y comprende los pronombres personales, la flexión verbal de persona, los posesivos y los demostrativos. La categoría gramatical de persona se refleja directamente en los diferentes papeles que los individuos desempeñan en el evento comunicativo: el hablante, el interlocutor, etc. (Levinson 2004: 112). En la misma terminología tradicional de la gramática, el término persona (primera, segunda y tercera), que procede del latín, indicaba una máscara y era la traducción de la palabra griega que identificaba al personaje dramático: el evento lingüístico era concebido como un drama en el que el papel principal correspondía a la primera persona, el papel secundario a la segunda y todos los demás a la tercera (Lyons 1977: 638). Puesto que en una conversación el centro deíctico (que Bühler 1934 denominó origo) coincide generalmente con el hablante, al cambiar el hablante también cambia el centro deíctico; de ahí que Jespersen (1922) y luego Jakobson (1957) utilizaran el término alternativo shifters para referirse a los elementos deícticos.

La deixis espacial localiza los elementos del contexto de la enunciación y comprende los demostrativos, determinados adverbios y frases preposicionales con significado locativo, así como algunos verbos de movimiento (Eguren 1999: 934). La deixis temporal sitúa lo descrito en el discurso en relación con el momento en que tiene lugar el evento comunicativo y comprende adverbios y frases nominales y preposicionales con valor temporal, además de la conjugación verbal de tiempo (Eguren 1999: 934). La deixis textual remite a fragmentos del texto que preceden o seguirán la enunciación de la expresión deíctica y que por eso pueden ser señalados. La deixis social refleja las relaciones sociales, en la lengua, con referencias directas u oblicuas al estatus social o al papel de los participantes en el evento comunicativo (Levinson 2004: 
119), como en el caso de las formas de tratamiento tú y usted en el español peninsular.

Además, según la naturaleza exclusivamente o no exclusivamente deíctica de una expresión, diferenciamos entre deixis pura y deixis impura (Eguren 1999: 935). Los deícticos puros tienen un significado de naturaleza exclusivamente deíctica y no añaden ningún otro significado (como los pronombres personales yo y tú). En cambio, los deícticos impuros vehiculan otra información, como el género en nosotras o el tiempo en la flexión verbal (soy/eral seré).

La deixis personal, de la que nos ocuparemos en este estudio, introduce una subjetividad imposible de erradicar en la estructura semántica de las lenguas naturales (Lyons 1977: 646) y desempeña un papel fundamental en la construcción retórica del discurso: a través de los deícticos personales el hablante se ubica a sí mismo en relación con los demás, se identifica como individuo o como parte de un grupo (con la primera persona del plural, tanto inclusiva como exclusiva). La mayoría de las veces, la identificación personal o grupal implica la oposición a otro grupo (vosotros/ustedes o ellos).

En este sentido, es posible dividir las expresiones deícticas en transparentes o completas por un lado y opacas o incompletas por el otro. Los deícticos transparentes o completos indican por sí mismos cuál es el referente que ha de tomarse en consideración en una situación de enunciación concreta. Ejemplos de deícticos personales transparentes son yo o tú. Eguren (1999: 935) enumera las siguientes características de los deícticos transparentes:

a) determinan a priori el tipo de referente denotado,

b) sólo pueden emplearse en un tipo de situación posible,

c) su referente no puede cambiarse por medio de un gesto, lo cual no quiere decir que no puedan ser reforzados por medio de gestos.

En cambio, los deícticos opacos o incompletos (él, ellas) no garantizan la identificación exacta de los referentes, admiten la referencia a diferentes elementos de la situación de enunciación y su referencia puede cambiar por medio de gestos. Eguren señala además que los deícticos opacos son los únicos que pueden emplearse anafóricamente.

La deixis personal, además, facilita la transmisión de convicciones políticas y contribuye a aceptar o rechazar lingüísticamente la responsabilidad de una acción, como indican las investigaciones sobre el discurso político o racista (Van Dijk 2003, 2008).

En consideración del papel que desempeñan las expresiones deícticas personales en el discurso, parece interesante estudiar el trato que reciben en los 
eventos comunicativos mediados por un intérprete. El objetivo de este trabajo es precisamente analizar la interpretación de las referencias deícticas personales (más específicamente, de la primera persona del singular y del plural) en un corpus de textos interpretados del español al italiano, para identificar posibles pautas interpretativas. ¿Qué cambios experimentan los elementos deícticos en la interpretación? ¿Se mantienen o se eliminan? ¿Existen tendencias interpretativas que caracterizan la interpretación del español al italiano?

\section{Material de investigación}

El corpus de referencia de nuestro estudio se compone de la transcripción de la versión original (VO, en italiano y en español) y la versión interpretada (VI, al italiano) de tres encuentros de un festival de periodismo que se celebra cada año en Ferrara y que organiza el semanal italiano Internazionale. En los encuentros suele haber un moderador italiano y uno o más invitados extranjeros, por lo general periodistas, que hablan de la situación política de su país o de algún fenómeno específico relacionado con su procedencia geográfica.

En eventos como los del festival, la identidad de los hablantes desempeña un papel fundamental. El festival se promociona como "un fin de semana con los periodistas de todo el mundo" (y no como "un fin de semana hablando del mundo"): el foco de los encuentros son los mismos periodistas extranjeros.

Los parámetros de la situación comunicativa son muy homogéneos en los tres encuentros: no hay variaciones en cuanto al setting, al iniciador y al organizador, a la técnica de interpretación (simultánea, en el caso de más de un invitado extranjero), a la caracterización sociológica de los intérpretes (profesionales de lengua italiana que trabajan en el mercado libre), a la lengua y la cultura de referencia de los participantes extranjeros (todos hispanohablantes que proceden de América Latina) y a la lengua y la cultura de referencia del público (italiano).

Nos parece interesante destacar que los organizadores del festival invitan cada año a periodistas de todo el mundo que hablan diferentes idiomas (inglés, francés, portugués, chino o ruso, entre otros): por eso, el análisis que llevaremos a cabo podría ampliarse a otras combinaciones lingüísticas (lengua extranjera-italiano) sin que variaran los demás parámetros de la situación comunicativa. Muchos encuentros están a disposición en las dos versiones (VO y VI) en la página que el semanal tiene en YouTube (www.youtube.com/ user/internazionale), que hemos consultado para nuestro estudio. Ampliar el análisis permitiría diferenciar entre los fenómenos que recurren en más de una combinación lingüística y las pautas que se presentan en una sola combinación. 
A continuación detallamos los títulos de los tres encuentros, las abreviaciones que utilizaremos para identificarlos en los párrafos sucesivos, las fechas y la duración (expresada en minutos) de cada evento:

\begin{tabular}{|l|c|c|c|}
\hline \multicolumn{1}{|c|}{ Título } & Abreviación & Fecha & Duración \\
\hline Colombia, una guerra senza colpevoli & CO & $01 / 10 / 11$ & 92 \\
\hline $\begin{array}{l}\text { Benvenuti a Narcolandia. Le nuove rotte del } \\
\text { traffico della droga }\end{array}$ & NA & $05 / 10 / 12$ & 113 \\
\hline Venezuela, la stella cadente & VE & $04 / 10 / 13$ & 86 \\
\hline
\end{tabular}

Tabla 1. Encuentros

En total, los invitados extranjeros de los tres encuentros son diez (cuatro mujeres y seis hombres); cuatro proceden de Colombia, tres de México, dos de Venezuela y uno de El Salvador. En los ejemplos se identificarán con las siguientes abreviaciones:

\begin{tabular}{|c|l|c|}
\hline Encuentro & \multicolumn{1}{|c|}{ Nombre } & Abreviación \\
\hline CO & Hollman Morris & HM \\
\hline CO & Javier Darío Restrepo & JDR \\
\hline CO & Alma Guillermoprieto & AG \\
\hline CO & Libardo Sarmiento Anzola & LSA \\
\hline NA & Carlos Dada & CD \\
\hline NA & Diego Enrique Osorno & DEO \\
\hline NA & Cynthia Rodríguez & CR \\
\hline VE & Boris Muñoz & BM \\
\hline VE & Maye Primera & MP \\
\hline VE & María Teresa Ronderos & MTR \\
\hline
\end{tabular}

Tabla 2. Participantes extranjeros

Como ya hemos destacado, el público es italiano y está formado en su gran mayoría por los lectores de la revista semanal Internazionale, que publica textos procedentes de periódicos de todo el mundo y traducidos al italiano (hay revistas parecidas en Francia, Courrier International, y en Portugal, Courrier Internacional). El servicio de interpretación corre a cargo de intérpretes profesionales que trabajan en el mercado libre italiano. En el caso de los encuentros analizados, todas las intérpretes son mujeres (cuyos nombres no se mencionan en la presentación de los encuentros en YouTube). Se trata de intérpretes de 
lengua materna italiana que poseen la combinación lingüística necesaria para interpretar directa e inversamente los encuentros (interpretación del español al italiano para el público e interpretación del italiano al español para los invitados extranjeros). Además, el festival cuenta con un servicio técnico que se ocupa de preparar las cabinas (móviles) y asegurar la calidad del sonido. Los tres encuentros se desarrollaron en el teatro municipal de Ferrara, con capacidad para 990 personas. Los auriculares disponibles para el público (con el canal de la interpretación del español al italiano) fueron cuatrocientos por encuentro.

La duración total de los encuentros es de 291 minutos. El total del texto transcrito es de 53975 palabras (28 392 de la VO, 25583 de la VI). En consideración del tipo de estudio llevado a cabo, hemos optado por una transcripción ortográfica.

Tomando en cuenta las dimensiones reducidas de nuestro corpus, nuestras conclusiones no pretenden tener valor general y constituyen una primera aproximación al tema; sin embargo, nos parece importante destacar que el corpus tiene la ventaja de presentar una gran homogeneidad en cuanto a los parámetros de la situación comunicativa.

\section{Análisis}

Desde un punto de vista lingüístico, la deixis personal se manifiesta a través de pronombres personales, tanto sujeto como complemento, de pronombres y adjetivos posesivos y de la flexión verbal. La mayoría de los textos que hablan de deixis personal indican que este tipo de deixis (en la forma transparente o completa) se expresa a través de la primera y de la segunda persona del singular y del plural; sin embargo, la variante lingüística que caracteriza a los periodistas latinoamericanos sustituye la segunda persona del plural por la tercera persona del plural (vosotros-ustedes), que se utiliza para dirigirse a los interlocutores.

En primer lugar, hemos identificado las marcas deícticas en el texto transcrito en español de la versión original (VO) y en la versión interpretada al italiano (VI). De este recuento hemos eliminado el marcador del discurso digamos/diciamo, por su cuantiosa presencia y por considerarlo vaciado de su significado deíctico.

\begin{tabular}{|l|l|l|l|}
\hline yo & $55 \%$ & io & $55 \%$ \\
\hline nosotros & $34 \%$ & noi & $37 \%$ \\
\hline tú & $5 \%$ & tu & $2 \%$ \\
\hline ustedes & $6 \%$ & voi & $6 \%$ \\
\hline
\end{tabular}

Tabla 3. Presencia porcentual de los deícticos en la VO y en la VI 
A primera vista, la relación porcentual de las referencias deícticas no varía mucho del texto español al texto italiano. Aunque la presencia cuantitativa de la deixis no presenta grandes variaciones entre la VO y la VI, la distribución de los deícticos en el texto italiano es el resultado de un proceso de interpretación que inevitablemente provoca cambios y modificaciones.

Considerando que en las dos versiones más de la mitad de las referencias deícticas conciernen a la primera persona singular y más de una cuarta parte a la primera persona plural, hemos decidido centrar el análisis en estas dos personas.

Analizando el corpus, hemos identificado cinco casos:

1) el referente deíctico se mantiene invariado en cuanto a la persona, aunque no necesariamente a la forma de expresarlo (por ejemplo, hemos considerado que no hay cambios en la deixis cuando se interpretó la expresión "yo creo" con "secondo me", porque ambos vehiculan la presencia de la primera persona del singular, sin diferenciar entre deixis pura o impura);

2) el referente deíctico cambia: por poner dos ejemplos, la primera persona del singular se convierte en primera persona del plural o en segunda persona del singular ( yo $\rightarrow$ noi o $t u$ ), la primera persona del plural se convierte en primera persona del singular (nosotros $\rightarrow$ io);

3) el referente deíctico desaparece;

4) se añade un referente deíctico donde no había ninguno;

5) en algunos (aunque pocos) casos, las intérpretes omiten un fragmento muy amplio del enunciado. Hemos decidido diferenciar entre la omisión del mero referente deíctico (caso 3) y la de un segmento de texto más amplio (caso 5).

Centraremos nuestro análisis en las referencias deícticas omitidas o añadidas de la primera persona del singular y del plural.

\subsection{Primera persona del singular}

Casi una cuarta parte de las referencias deícticas (24,21\% del total) se omite en la VI. Al analizar las referencias desaparecidas, notamos que buena parte de ellas son cláusulas matrices introducidas por el verbo creo que se utilizan para mitigar la fuerza de una aserción y reiterar la subjetividad del hablante, destacando el yo como centro deíctico activo (Haverkate 1998: 48; Barrenechea 1979). A continuación ofrecemos una pequeña muestra de las ocurrencias encontradas en el corpus: 


\begin{tabular}{|c|c|c|l|l|}
\hline Encuentro & Nombre & Minuto & \multicolumn{1}{|c|}{ VO } & \multicolumn{1}{|c|}{ VI } \\
\hline NA & CR & 47.23 & $\begin{array}{l}\text { el número yo creo que es } \\
\text { incalculable }\end{array}$ & $\begin{array}{l}\text { è incalcolabile il numero } \\
\text { delle vittime }\end{array}$ \\
\hline VE & BM & 10.38 & $\begin{array}{l}\text { ese es el telón de fondo } \\
\text { económico que creo } \\
\text { que ayuda a explicar esa } \\
\text { dramática caída en los } \\
\text { números de aceptación de } \\
\text { la gestión de Maduro }\end{array}$ & $\begin{array}{l}\text { questo è lo sfondo } \\
\text { economico la cornice } \\
\text { entro cui si devono } \\
\text { interpretare tutte le cifre } \\
\text { di cui abbiamo parlato per } \\
\text { Nicolás Maduro }\end{array}$ \\
\hline VE & MP & 01.08 .00 & $\begin{array}{l}\text { plantear la división } \\
\text { política en Venezuela } \\
\text { como una lucha de clase } \\
\text { o como un problema } \\
\text { ideológico yo creo que es } \\
\text { equivocado no }\end{array}$ & $\begin{array}{l}\text { parlare di divisione } \\
\text { politica in Venezuela } \\
\text { come lotta di classe come } \\
\text { problema ideologico è un } \\
\text { errore }\end{array}$ \\
\hline CO & LSA & 01.22 .40 & $\begin{array}{l}\text { el botín de la guerra } \\
\text { de robo en tierras a } \\
\text { campesinos es de 7 } \\
\text { millones de hectáreas creo } \\
\text { que una extensión muy } \\
\text { grande }\end{array}$ & $\begin{array}{l}\text { sette milioni di ettari sono } \\
\text { stati rubati ai contadini } \\
\text { quindi una superficie } \\
\text { enorme }\end{array}$ \\
\hline CO & JDR & 01.18 .02 & $\begin{array}{l}\text { pero en este momento la } \\
\text { situación del país creo que } \\
\text { se puede resumir en una } \\
\text { gran expectativa }\end{array}$ & $\begin{array}{l}\text { ma in questo momento la } \\
\text { situazione del paese può } \\
\text { essere riassunta in una } \\
\text { grande aspettativa }\end{array}$ \\
\hline
\end{tabular}

Tabla 4. Deícticos omitidos (primera persona del singular)

A veces, la atenuación de la fuerza del enunciado pasa al adverbio forse ('quizás'):

\begin{tabular}{|c|c|l|l|l|}
\hline Encuentro & Nombre & Minuto & \multicolumn{1}{c|}{ VO } & \multicolumn{1}{c|}{ VI } \\
\hline CO & LSA & 36.40 & $\begin{array}{l}\text { yo creo que con Colombia } \\
\text { pasa un poco lo mismo }\end{array}$ & $\begin{array}{l}\text { ecco in Colombia forse } \\
\text { accade un po' lo stesso }\end{array}$ \\
\hline CO & LSA & 01.17 .30 & $\begin{array}{l}\text { hacer que en unas } \\
\text { elecciones no sé si de } \\
\text { estas u otras que vendrán } \\
\text { más allá se pueda eliminar } \\
\text { una casta política }\end{array}$ & $\begin{array}{l}\text { far sì che nel corso di } \\
\text { un'elezione libera forse } \\
\text { non questa forse una } \\
\text { prossima sia possibile } \\
\text { eliminare una casta } \\
\text { politica }\end{array}$ \\
\hline
\end{tabular}

Tabla 5. Sustitución deíctico - adverbio forse

En cambio, las referencias deícticas que se añaden son menores: corresponden al $16,77 \%$ del total, y son el fruto de una elección de las intérpretes. La 
mayoría de los deícticos añadidos tienen la función de marcador discursivo y se deben a la mínima posibilidad de planificar el discurso, tal como dicevo u otros verbos que expresan la acción del decir, como ilustran los siguientes ejemplos extraídos del corpus:

\begin{tabular}{|c|c|c|l|l|}
\hline Encuentro & Nombre & Minuto & \multicolumn{1}{|c|}{ VO } & \multicolumn{1}{|c|}{ VI } \\
\hline CO & HM & 19.40 & la historia nos enseña & la storia dicevo ci insegna \\
\hline CO & LSA & 50.22 & $\begin{array}{l}\text { ese último periodo ha } \\
\text { implicado una guerra }\end{array}$ & $\begin{array}{l}\text { e in quest'ultimo periodo } \\
\text { dicevo c'è stata una guerra }\end{array}$ \\
\hline CO & LSA & 47.16 & $\begin{array}{l}\text { Colombia es uno los países } \\
\text { por los beneficios que le } \\
\text { da a las transnacionales } \\
\text { yal sector financiero } \\
\text { internacional una de } \\
\text { las utilidades más altas } \\
\text { que puede uno mirar de } \\
\text { rentabilidad de negocios en } \\
\text { el mundo }\end{array}$ & $\begin{array}{l}\text { la Colombia è uno dei } \\
\text { paesi per quanto riguarda } \\
\text { il settore finanziario } \\
\text { nazionale dicevo è uno dei } \\
\text { paesi che ha la redditività } \\
\text { maggiore per quanto } \\
\text { riguarda l'estero }\end{array}$ \\
\hline CO & LSA & 47.42 & $\begin{array}{l}\text { qué quiere decir eso que } \\
\text { la rentabilidad de una } \\
\text { transnacional operando en } \\
\text { Colombia es del 33\% }\end{array}$ & $\begin{array}{l}\text { perché vi dico tutto questo } \\
\text { perché semplicemente per } \\
\text { dire che la redditività di } \\
\text { una multinazionale che } \\
\text { lavora in Colombia è del 33 } \\
\text { per cento }\end{array}$ \\
\hline CO & AG & 58.55 & $\begin{array}{l}\text { una de ellas ha sido una de } \\
\text { las ciudades más afectadas } \\
\text { por la violencia que fue } \\
\text { Medellín afectada por la } \\
\text { violencia del narcotráfico }\end{array}$ & $\begin{array}{l}\text { e curiosamente uno di } \\
\text { essi una delle città più } \\
\text { toccante dalla violenza del } \\
\text { narcotraffico e parlo di } \\
\text { Medellín }\end{array}$ \\
\hline
\end{tabular}

Tabla 6. Deícticos añadidos (primera persona del singular)

Destacamos un caso en el que la atenuación de la fuerza del enunciado vehiculada por el tiempo condicional (habría) pasa a un verbo (credo) que, además de tener la misma función de atenuación, introduce la dimensión deíctica de la primera persona singular:

\begin{tabular}{|c|c|c|l|l|}
\hline Encuentro & Nombre & Minuto & \multicolumn{1}{|c|}{ VO } & \multicolumn{1}{c|}{ VI } \\
\hline NA & CD & 21.32 & $\begin{array}{l}\text { lo primero que habría que } \\
\text { establecer como punto de } \\
\text { partida }\end{array}$ & $\begin{array}{l}\text { credo che la prima cosa da } \\
\text { dire come punto di partenza }\end{array}$ \\
\hline
\end{tabular}

Tabla 7. Sustitución condicional - deíctico (primera persona singular) 


\subsection{Primera persona del plural}

$\mathrm{Al}$ analizar la omisión de las referencias deícticas de la primera persona plural, notamos que en muchos casos la primera persona del plural se convierte en una tercera persona del plural en presencia de una estructura sintáctica específica: sustantivo plural + verbo en primera persona del plural. Se trata de una construcción sintáctica que en español identifica de forma más directa al hablante como parte de un grupo, como en la frase "los españoles somos menos altos que los ingleses" (Carrera Díaz 1997: 36). En español un verbo de primera persona del plural puede ser precedido por un nombre genérico, mientras que en italiano la misma estructura resulta agramatical, puesto que un nombre genérico plural requiere necesariamente un verbo en tercera persona del plural. Por el carácter lineal del lenguaje y la velocidad de elocución, las intérpretes se encuentran ante una estructura difícil de interpretar con una primera persona del plural. En algunos casos, las intérpretes intentan reintroducir el elemento deíctico dando lugar a anacolutos:

\begin{tabular}{|c|l|l|l|l|}
\hline Encuentro & Nombre & Minuto & \multicolumn{1}{|c|}{ VO } & \multicolumn{1}{c|}{ VI } \\
\hline CO & HM & 17.19 & $\begin{array}{l}\text { Quisieron pretendieron } \\
\text { que los periodistas que } \\
\text { durante muchos y muchos } \\
\text { años hemos cubierto } \\
\text { la historia del conflicto } \\
\text { armado en Colombia nos } \\
\text { quedáramos en las salas } \\
\text { de redacción es decir } \\
\text { nos quedáramos en las } \\
\text { sombras. }\end{array}$ & $\begin{array}{l}\text { Hanno voluto fare in } \\
\text { modo che i giornalisti che } \\
\text { durante molti moltissimi } \\
\text { anni si sono occupati } \\
\text { di coprire la storia del } \\
\text { conflitto armato in } \\
\text { Colombia rimanessero } \\
\text { fuori dal nucleo ovvero } \\
\text { volevo volevano che } \\
\text { rimanessimo noi } \\
\text { nell'ombra }\end{array}$ \\
\hline CO & HM & 25.46 & $\begin{array}{l}\text { diciéndoles a los } \\
\text { periodistas que } \\
\text { entrábamos a las zonas } \\
\text { de conflicto aliado del } \\
\text { terrorismo }\end{array}$ & $\begin{array}{l}\text { dicendo ai giornalisti } \\
\text { che entravano nelle zone } \\
\text { di conflitto che eravamo } \\
\text { alleati del terrorismo }\end{array}$ \\
\hline NA & CR & $\begin{array}{l}\text { todos estos fenómenos } \\
\text { fenómenos políticos } \\
\text { obviamente que también } \\
\text { tienen tratos con } \\
\text { narcotraficantes son } \\
\text { las cosas que tienen } \\
\text { que han hecho que los } \\
\text { ciudadanos no tengamos } \\
\text { ninguna confianza en las } \\
\text { autoridades }\end{array}$ & $\begin{array}{l}\text { tutti questi fenomeni } \\
\text { politici che sono legati al } \\
\text { narcotraffico sono ciò che } \\
\text { ha fatto sì che i cittadini } \\
\text { non abbiano più nessuna } \\
\text { fiducia nelle autorità }\end{array}$ \\
\hline
\end{tabular}

Tabla 8. Deícticos omitidos (primera persona del plural) 
En cuanto a las referencias deícticas añadidas, notamos que se consolida la identidad de grupo en relación a la procedencia geográfica de los hablantes, que es uno de los rasgos más evidentes que diferencian el público (italiano) de los invitados (extranjeros):

\begin{tabular}{|c|c|c|l|l|}
\hline Encuentro & Nombre & Minuto & \multicolumn{1}{|c|}{ VO } & \multicolumn{1}{c|}{ VI } \\
\hline VE & BM & 09.28 & $\begin{array}{l}\text { Venezuela sufre una crisis } \\
\text { de abastecimiento de } \\
\text { bienes esenciales }\end{array}$ & $\begin{array}{l}\text { credo che tutti in sala } \\
\text { sappiano che in Venezuela } \\
\text { abbiamo una crisi nel } \\
\text { reperimento dei beni di } \\
\text { base }\end{array}$ \\
\hline VE & MP & 20.37 & $\begin{array}{l}\text { Venezuela en este } \\
\text { momento está importando } \\
\text { gasolina }\end{array}$ & $\begin{array}{l}\text { adesso stiamo comprando } \\
\text { combustibile }\end{array}$ \\
\hline VE & MP & 24.25 & $\begin{array}{l}\text { justamente por el sistema } \\
\text { económico tan perverso } \\
\text { que se tiene ahora que es } \\
\text { que en Venezuela }\end{array}$ & $\begin{array}{l}\text { a causa del sistema } \\
\text { economico perverso in cui } \\
\text { viviamo attualmente in } \\
\text { Venezuela }\end{array}$ \\
\hline
\end{tabular}

Tabla 9. Deícticos añadidos (primera persona del plural)

Destacamos, además, la transformación de una referencia deíctica del plano espacial (acá) al plano personal (noi), manteniendo invariado el centro deíctico:

\begin{tabular}{|c|c|c|l|l|}
\hline Encuentro & Nombre & Minuto & \multicolumn{1}{c|}{ Versión original } & \multicolumn{1}{c|}{ Versión interpretada } \\
\hline VE & MP & 01.07 .58 & $\begin{array}{l}\text { no ocurre como en } \\
\text { Colombia que desde hace } \\
\text { más o menos doscientos } \\
\text { años gobiernan las mismas } \\
\text { familias acá es como que } \\
\text { cada cincuenta años se } \\
\text { establece un nuevo grupo } \\
\text { de poder }\end{array}$ & $\begin{array}{l}\text { non succede come in } \\
\text { Colombia dove diciamo } \\
\text { da duecento anni } \\
\text { praticamente governa la } \\
\text { stessa famiglia da noi ogni } \\
\text { cinquant'anni si consolida } \\
\text { un nuovo gruppo al } \\
\text { potere }\end{array}$ \\
\hline
\end{tabular}

Tabla 10. Sustitución deíctico espacial - deíctico personal

\subsection{La relativización de la deixis}

Por último, quisiéramos hacer algunas observaciones sobre la deixis personal utilizada en relación con una narración en la que el hablante se identifica con otro hablante: como indicó Bühler (1934), las expresiones deícticas pueden transponerse o relativizarse en relación con otro origo, por lo general con la persona del protagonista en el espacio y en el tiempo relevante de 
una narrativa (Fillmore 1975). A menudo, la citación es introducida por un elemento, denominado marco o marco citante, como un verbo del decir. En ese caso, el hablante utiliza deícticos que no se orientan hacia su proprio origo, sino hacia otro. El centro deíctico de los enunciados no se modifica en comparación con la producción originaria: el hablante cede su voz a una persona distinta, que actúa como el hablante en el discurso referido. A continuación presentamos un ejemplo de este fenómeno sacado del encuentro sobre Venezuela. A través del verbo dijo, María Teresa Rondero introduce en dos ocasiones un origo ficticio (el gobierno y la guerrilla) al que cede su voz con el uso de la primera persona del singular (no me siento). Notamos que en la VI se ha añadido el pronombre personal yo, ausente en la VI, que además se marca con un acento prosódico, probablemente para destacar aún más la naturaleza ficticia del origo:

\begin{tabular}{|c|c|c|l|l|}
\hline Encuentro & Nombre & Minuto & \multicolumn{1}{c|}{ Versión original } & \multicolumn{1}{c|}{ Versión interpretada } \\
\hline VE & MTR & 30.27 & $\begin{array}{l}\text { tiene cinco puntos la } \\
\text { agenda dos puntos que } \\
\text { puso el gobierno y que dijo } \\
\text { no me siento a negociar } \\
\text { sin estos dos puntos dos } \\
\text { puntos que puso la guerrilla } \\
\text { que dijo no me siento } \\
\text { a negociar sin estos dos } \\
\text { puntos }\end{array}$ & $\begin{array}{l}\text { ci sono cinque punti sul } \\
\text { programma due imposti } \\
\text { dal governo che ha detto io } \\
\text { non mi siedo a negoziare } \\
\text { se non accadono queste due } \\
\text { cose due punti stabiliti dai } \\
\text { guerriglieri che hanno detto } \\
\text { io non mi siedo al tavolo } \\
\text { negoziale senza questi due } \\
\text { punti }\end{array}$ \\
\hline
\end{tabular}

Tabla 11. Origo ficticio

\section{Conclusiones}

El estudio del corpus revela que, aunque a primera vista la relación porcentual de las referencias deícticas es relativamente similar en la VO y en la VI, los textos de la VI experimentan diferentes cambios: algunas referencias se omiten, mientras que otras se añaden o se modifican. Los cambios en la distribución de la deixis en la VI conllevan una modificación parcial del mensaje de la VO. En el caso de la primera persona del singular, los deícticos que desaparecen de la VI son sobre todo verbos de opinión y expresiones que mitigan la fuerza de los enunciados. Dichas omisiones disminuyen, aunque sea parcialmente, la subjetividad de los hablantes en la VI frente a la VO y confieren a los textos de la VI un tono más objetivo. En cambio, los deícticos añadidos son sobre todo marcadores discursivos que responden a la mínima posibilidad de planificar el texto por parte de las intérpretes, debido a la linealidad de la VO y 
a la velocidad de elocución. Su función no corresponde a la de las referencias deícticas omitidas y su presencia no compensa la disminución de la subjetividad de los hablantes en la VI.

En cuanto a la primera persona del plural, algunas referencias deícticas desaparecen a causa de una asimetría sintáctica entre el español y el italiano que resulta difícil de gestionar en la interpretación, una vez más por la linealidad y la velocidad de elocución, combinadas con un décalage limitado. Como en el caso de la primera persona del singular, la omisión de las referencias deícticas de la primera persona del plural reduce la subjetividad de los hablantes en la VI y disminuye su implicación en el discurso. Por último, y siempre en cuanto a la primera persona del plural, se añaden referencias que consolidan la identidad de los hablantes en relación con su procedencia geográfica, una elección que nos parece acertada considerando el contexto socio-comunicativo del festival de Internazionale que, como mencionábamos al principio, se presenta como un fin de semana con los periodistas "de todo el mundo".

\section{Referencias bibliográficas}

BARRENECHEA, Ana María. (1979) "Operadores pragmáticos de actitud oracional. Los adverbios en -mente y otros signos". En: Barrenechea, Ana María et al. (eds.) Estudios lingüísticos y dialectológicos: Temas hispánicos. Buenos Aires: Hachette, pp. 39-59.

BAzZANella, Carla. (1994) Le facce del parlare. Florencia: La Nuova Italia.

BüHLER, Karl. (1934) Sprachteorie. Die Darstellungfunktion der Sprache. Stuttgart:

Gustav Fischer. Citado por la traducción italiana de Serena Cattaruzza

Derossi: Teoria del linguaggio. La funzione rappresentativa del linguaggio. Roma: Armando, 1983.

CARRERA DíAZ, Manuel. (1997) Grammatica spagnola. Bari/Roma: Laterza.

EGUREN, Luis. (1999) "Pronombres y adverbios demostrativos". En: Bosque, Ignacio \& Violeta Demonte (eds.) Gramática descriptiva de la lengua española. Madrid: Espasa-Calpe, vol. 1, pp. 1275-1316.

Fillmore, Charles. (1975) Santa Cruz Lectures on Deixis, 1971. Bloomington: Indiana University Linguistic Club.

HAVERKATE. Hank. (1998) "Estrategias de cortesía. Análisis intercultural." En: Celis, Ángela \& José Ramón Heredia (eds.) Actas del VII Congreso de ASELE. Lengua y cultura en la enseñanza de español a extranjeros. Cuenca: Ediciones de la Universidad de Castilla-La Mancha, pp. 45-57.

JAKOBSON, Roman. (1957) "Shifters, verbal categories, and the Russian verb". En: Waugh, L. \& M. Monville-Burston (eds.) 1990. On Language: Roman Jakobson. Cambridge (Mass.): Harvard University Russian Language Project, pp. 386-392. 
JeSPERSEN, Otto. (1922) Language. Its Nature, Development and Origin. London: George Allen \& Unwin.

Levinson, Stephen C. (1983) Pragmatics. Cambridge: Cambridge University Press.

LeVinson, Stephen C. (2004) "Deixis". En: Horn, Laurence \& Gregory Ward

(eds.). The Handbook of Pragmatics. Oxford: Blackwell, pp. 97-121.

LyONS, John. (1977) Semantics. Vol. 2. Cambridge: Cambridge University Press.

Citado por la traducción española: Semántica. Barcelona: Teide, 1980.

RoMmETVEIT, Ragnar. (1968) Words, meanings and messages. New York: Academic Press.

VANelli, Laura \& Lorenzo Renzi. (2001) "La deissi". En: Renzi, Lorenzo; Giampaolo Salvi \& Anna Cardinaletti (eds.) Grande grammatica italiana di consultazione. Bolonia: Il Mulino, vol. 3, pp. 261-350.

VAN DijK, Teun. (2008) Discurso y poder. Barcelona: Gedisa.

VAN DijK, Teun. (2003) Racismo y discurso de las élites. Barcelona: Gedisa.

\section{NOTA BIOGRÁFICA / BIONOTE}

SARA BANI se doctoró en Ciencia de la Traducción por la Universidad de Bolonia en 2007. Actualmente es investigadora de Lengua española y traducción (RTD, L-LIN/07) en la Universidad de Catania. Entre sus principales intereses de investigación se encuentran el discurso periodístico y su traducción, la lexicografía, la mediación lingüística y la interpretación.

SARA BANI received her PhD in Translation Science from the University of Bologna in 2007. She is currently a researcher in Spanish language and literature (RTD, L-LIN/07) at the University of Catania. Her main research interests include journalistic discourse and translation, lexicography, linguistic mediation and interpretation. 